\begin{tabular}{|c|c|c|}
\hline & Parasomnie & Epilepsie (fokal) \\
\hline Beginn & Kindheit & jedes Alter \\
\hline Dauer & $1-10 \mathrm{~min}$ & $0,5-4 \min$ \\
\hline Semiologie & bunt & uniform, stereotyp \\
\hline Ablauf & $\begin{array}{l}\text { komplex, unter Um- } \\
\text { ständen mit gezieltem } \\
\text { Handeln }\end{array}$ & $\begin{array}{l}\text { simple, meist mit un- } \\
\text { gezieltem Handeln }\end{array}$ \\
\hline Erinnerung & $\begin{array}{l}\text { unter Umständen an } \\
\text { Traum }\end{array}$ & keine/eventuell Aura \\
\hline Einbettung & $\begin{array}{l}\text { aus Schlaf; Patient } \\
\text { (schwer) weckbar, tritt } \\
\text { sporadisch auf } \\
\text { danach: Schlaf }\end{array}$ & $\begin{array}{l}\text { aus Schlaf- oder Wach- } \\
\text { phase, Patient nicht } \\
\text { "weckbar", tritt in Clus- } \\
\text { tern auf, danach: wach }\end{array}$ \\
\hline
\end{tabular}

ne - bei sehr ähnlichem Ablauf der Verhaltensschablonen - ein Langzeit-EEG-Monitoring und gegebenenfalls eine Polysomnografie notwendig sein.

\section{"Central Motor Pattern" als Ursache beider Störungen?}

Zentrale Verhaltensschablonen (Central Motor Patterns) als Reaktion auf Bedrohung, Angst oder auch Sex, die früh in der Phylogenese entstanden sind und als erlerntes Verhalten bis heute persistieren, können ursächlich für die ähnlichen Verhaltensautomatismen sein. Denn beide Störungen führen im Schlaf zu einer Aktivierung der gleichen Strukturen: Bei Parasomnien kommt es zu einem lokalen Teilerwachen, bei Epilepsie zu einer lokalen elektrischen Hyperaktivität frontolimbischer Strukturen. „In beiden Fällen resultiert eine Prädominanz der frontolimbischen Aktivität und somit eine Manifestation der dort gelagerten Handlungsschablonen, die sich ungehindert austoben können“, erklärte Voges.

Michael Koczorek

Symposium "Sexolepsie oder Sexsomnie? Anfälle mit sexuellen Verhaltensschablonen im Rahmen von Parasomnie oder Epilepsie“; 25. Kongress der Deutschen Gesellschaft für Schlafforschung und Schlafmedizin, Münster, 9.11.2017

\title{
RLS: Augmentation frühzeitig erkennen
}

\begin{abstract}
Augmentation ist ein häufiges Phänomen im Therapieverlauf eines Restless-Legs-Syndroms, das früh erkannt werden sollte. Die therapeutischen Optionen sind jetzt erweitert worden.
\end{abstract}

Diagnosekriterien des Restless-Legs-Syndroms (RLS) betreffen den Bewegungsdrang, der meist mit Missempfindungen in den Beinen, seltener auch den Armen einhergeht, das Auftreten bei körperlicher Inaktivität, die Besserung durch Bewegung (solange diese anhält) und - vor Beginn der Erkrankung beziehungsweise vor Langzeitbehandlung mit Dopaminagonisten - ein zirkadianer Verlauf mit Verschlechterung am Abend. „Diese vier Minimalkriterien sind hilfreich und führen in den meisten Fällen zu einer erfolgreichen Diagnose", konstatierte Professorin Birgit Högl, Leiterin des Schlaflabors und der Spezialambulanz für Schlafstörungen an der Universitätsklinik für Neurologie der Medizinischen Universität Innsbruck.

\section{Kriterien zur Diagnose}

Seit 2014 wird nun ein fünftes Kriterium gefordert: der aktive Ausschluss von „Mimics“ als Differenzialdiagnosen, die die Symptome eines RLS imitieren können [Allen RP et al. Sleep Medicine 2014; 15 (8): 860 - 73]. Dazu gehören alle Erkrankungen, die Symptome in den Beinen verursachen können - von Polyneuropathie und Myalgie, über Venenleiden und Ödeme bis zu arthritischen Beschwerden. Grund für das Update der Diagnosekriterien waren Studiendaten, die zeigten, dass es bei ausschließlicher Berücksichtigung der vier Kriterien in 16\% der Fälle zu falsch positiven Ergebnissen kam [Hening WA et al. Sleep Medicine 2009; 19 (9): 976 - 81].

In den vergangenen Jahren stellte sich heraus, dass sich im Verlauf der Therapie mit dopaminergen Medikamenten weit häufiger als bislang angenommen eine Augmentation entwickelt und die Periode der Wirksamkeit limitiert ist. Frühe Zeichen sollten an eine solche Verschlechterung des RLS denken lassen [Garcia-Boreguerro D et al. Sleep Med 2016; $21: 1$ - 11]: So treten die Symptome früher am Tag auf als zu Beginn der Behandlung, die Latenz bis zum Auftreten in Ruhe wird kürzer oder die Symptome breiten sich auf andere Körperteile aus. Ein weiteres Zeichen ist die paradoxe Response mit Verschlechterung oder zeitweise Verbesserung der Symptomatik bei Erhöhung oder Reduktion der Medikamentendosis.

\section{Medikamentöse Therapie in der Praxis}

Zur Prävention der Augmentation sollte eine medikamentöse Therapie nur bei starken Beschwerden gestartet werden. Für Dopaminagonisten ist die niedrigste wirksame Dosis anzuwenden oder es sollten bereits initial Alpha-2-delta-Liganden wie Pregabalin oder Gabapentin eingesetzt werden, die ein geringes Augmentationsrisiko aufweisen. Retardierte stark wirksame Opioide wie Oxycodon/Naloxon können bei schwerem, behandlungsresistentem RLS verordnet werden. Für intravenöse EisenCarboxymaltose wurde unabhängig von einem Eisenmangel ebenso wie für pneumatische Kompressionsdevices ein Effekt gezeigt. Vitamin C und E sowie Training können bei urämischem RLS wirksam sein.

Michael Koczorek

Symposium "Update zum Restless Legs Syndrom und anderen Bewegungsstörungen im Schlaf", 25. Kongress der Deutschen Gesellschaft für Schlafforschung und Schlafmedizin, Münster, 9.11.2017 